\title{
EFICIENCIA DE LAS MISIONES SOCIALES DE EDUCACIÓN UTILIZANDO LÓGICA DIFUSA
}

Darwin Diskey Contreras-Guillén

Universidad de los Andes

Venezuela

Alberto José Hurtado-Briceño

Universidad de los Andes

Venezuela 
Panorama Económico, Vol. 25 - No.1(Enero - Marzo de 2017), pp. 19-42

Darwin Diskey Contreras-Guillén

Alberto José Hurtado-Briceño

\title{
Eficiencia de las misiones sociales de educación utilizando lógica difusa
}

\begin{abstract}
Resumen
Esta investigación tuvo como objetivo determinar un modelo matemático de eficiencia de las misiones sociales dirigidas a la educación en el Área Metropolitana del Estado de Mérida mediante la aplicación de lógica difusa para la medición integral del fenómeno en estudio, lográndose explicar la eficiencia de las misiones educativas desde un enfoque multidimensional. La metodología de tipo descriptivo-exploratorio, permitió describir y explicar variables no contempladas en métodos tradicionales de medición de eficiencia de políticas sociales. En la investigación, las unidades de objeto de observación y estudio fueron los beneficiarios o participantes de cada una de las misiones sociales educativas (Robinson, Ribas, y Sucre), mediante una muestra de 330 personas, significativa de la población. Se concluyó que durante el periodo de estudio (2013-2015), las misiones educativas obtuvieron un umbral de eficiencia descrito por: [0.263, 0.329], $[0.291,0.355],[0.254,0.324]$ respectivamente, que representa una clasificación de casi ineficiente a cercana a ineficiente.

Palabras clave: misiones educativas, lógica difusa, eficiencia, políticas públicas, evaluación de políticas sociales.
\end{abstract}

Clasificación JEL: I28, I38, C45

\section{Efficiency of education social missions using fuzzy logic}

\begin{abstract}
This research had as objective to determine a mathematical model of efficiency of the social missions directed to the education in the Metropolitan Area of Mérida through the fuzzy logic method for the measurement of the phenomenon under study, achieving to explain the efficiency of the educational missions from a multidimensional approach. The research carried out presents a descriptive-exploratory methodology, which allowed describing and explaining variables not included in the traditional methods of measuring social policy efficiency. In the research, the units of observation were the beneficiaries or participants of each of the social educational missions (Robinson, Ribas, Sucre), through a sample of 330 people. It was concluded that educational missions obtained an efficiency threshold described by: [0.263, 0.329], [0.291, 0.355], [0.254, 0.324] respectively, representing a classification of almost inefficient to close to inefficient.
\end{abstract}

Keywords: educational missions, fuzzy logic, efficiency, public policies, evaluation of social policies. JEL Classification: I28, I38, C45

\section{Efficience des missions sociales d'education en utilisant la logique floue}

\begin{abstract}
Résumé
L'objectif de cette recherche était de déterminer un modèle mathématique de l'efficacité des missions sociales visant l'éducation dans la région métropolitaine de l'État de Mérida, au moyen d'une logique floue. L'application de la logique floue a permis l'utilisation de variables objectives et subjectives pour la mesure intégrale du phénomène à l'étude, en permettant d'expliquer l'efficacité des missions éducatives à partir d'une approche multidimensionnelle. La recherche effectuée présente une méthodologie de type descriptive-exploratoire, qui a permis de décrire et d'expliquer des variables ignorées dans les méthodes traditionnelles de mesure de l'efficacité des politiques sociales. Dans l'enquête, les unités d'observation et d'étude étaient les bénéficiaires ou les participants de chacune des missions sociales éducatives (Robinson, Ribas et Sucre). Nous avons travaillé sur un échantillon de 330 personnes, ce qui représente un nombre important de la population pour élaborer les résultats. Il a été conclu qu'au cours de la période étudiée 2013-2015, les missions éducatives avaient atteint un seuil d'efficacité décrit par: [0.263, 0.329], [0.291, 0.355], $[0.254,0.324]$ respectivement, ce qui correspond à une classification presque inefficace presque inefficace. Mots-clés: Missions pédagogiques, logique diffuse, efficacité, politiques publiques, évaluation des politiques sociales.
\end{abstract}

Nomenclature JEL: I28, I38, C45 


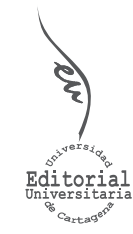

Artículo de Investigación

\section{Eficiencia de las misiones sociales de educación utilizando lógica difusa}

INFORMACIÓN DEL ARTíCULO

Recepción de artículo: 22/10/2016

Concepto de evaluación: 15/11/2016

Aceptación de artículo: 21/12/2016
Darwin Diskey Contreras-Guillén* Universidad de los Andes

Venezuela

Alberto José Hurtado-Briceño Universidad de los Andes Venezuela

\section{INTRODUCCIÓN}

Las misiones educativas forman parte de las misiones sociales iniciadas entre los años 2003-2004 durante el primer gobierno del presidente Chávez. Se plantearon como políticas sociales administradas por el gobierno central para atender las necesidades y dificultades de la población menos favorecida de Venezuela (Hurtado y Zerpa, 2016). Entre las creadas para el área educativa se encuentran la Misión Robinson I y II, la Misión Ribas y la Misión Sucre. Sobre estas misiones descansa gran parte de la promoción de los logros de la gestión de gobierno, ya que obtuvieron el mayor alcance a nivel territorial y poblacional (D’Elias y Cabeza, 2008). Por otro lado, las misiones en educación, salud y alimentación consiguieron captar el interés nacional e internacional por encontrar modelos de políticas públicas emergentes de inclusión social y de atención a los déficits de acceso a servicios sociales en las zonas urbanas más pobres, específicamente el abastecimiento de alimentos, la atención primaria de la salud y la inserción educativa.

Las misiones sociales se pueden considerar como una nueva manera de nombrar y entender las nociones de política social, desde el anuncio de su creación hasta la actualidad, han jugado un papel clave en lo que es la gestión social del gobierno. Como lo plantea Gómez (2007) a dichas misiones se han dedicado grandes inversiones de recursos financieros, materiales y humanos, en un esfuerzo por ofrecer respuestas a un gran número de venezolanos en situación de pobreza que demandan soluciones en materia de acceso al sistema educativo, empleo y organización de carácter productivo, entre otras. Es de esperar que este tipo de intervención social genere muchas interrogantes

\footnotetext{
* Autor para correspondencia

Correos electrónicos: darwindcg@gmail.com*, ajhurtado@ula.ve
} 
en cuanto a su operatividad, fuentes de financiamiento y dinámica en general, más aún cuando se desarrollan de una forma distinta a lo que tradicionalmente ha sido el funcionamiento del sistema de bienestar social en Venezuela. En este sentido es fundamental profundizar el debate de este tema, fundamentado en la evaluación de los alcances de este tipo de política social. Esencialmente porque se ha evidenciado que a la par de que se intentan resolver de manera inclusiva los déficits sociales, se refuerzan viejos problemas de la política pública, tales como, la ineficiencia del gasto social, la falta de articulación entre política económica y política social, la ausencia de racionalidad técnica y financiera entre otros.

La eficiencia de un programa social es compleja de evaluar, la razón es simple, la medida de eficiencia puede abordarse desde diferentes perspectivas y tiene múltiples posibles soluciones. Por esta razón, para hablar de eficiencia de la forma más objetiva posible, se debe primero definir qué se puede entender por eficiencia, segundo especificar cómo se evaluará esa eficiencia, y tercero dejar claro qué nivel de eficiencia se desea y si puede ser alcanzado. En la presente investigación el término eficiencia se entendió como el cociente entre salidas y entradas, es decir, la relación entre los resultados obtenidos en función de los recursos utilizados y respecto a las metas planteadas, lo cual permitió valorar hasta qué punto los recursos utilizados han permitido resolver el problema básico que atiende la política social orientada al sector educativo, teniendo en cuenta su impacto económico y social en el bienestar de la población. Evaluar la eficiencia para determinar el éxito o fracaso de las misiones sociales en educación.
De esta manera, como las misiones sociales de educación son programas públicos con muchos objetivos y desde su puesta en práctica no han sido evaluadas en términos de calidad y eficiencia, se plantea hacer uso de la lógica difusa para la determinación de un modelo matemático que permita explicar la eficiencia de las misiones educativas en el Área Metropolitana del estado Mérida, Venezuela. En este contexto, se considera la lógica difusa como un método alternativo que permite tomar en cuenta el nivel de incertidumbre de las variables que evalúa (Zadeh, 1965), ya que el razonamiento y pensamiento humano frecuentemente conlleva información vaga, imprecisa, incierta, y ambigua (debido a la inexactitud inherente de los conceptos humanos y del razonamiento basado en experiencias similares), se espera, mediante la aplicación de este método tomar opiniones de parte de los participantes de las misiones educativas para conocer y evaluar su eficiencia como políticas sociales.

\section{Herramientas de Lógica difusa}

\section{Expertizaje}

El expertizaje representa la herramienta que permite hacer la evaluación a la opinión de expertos en función de un determinado tema transformándola en un lenguaje científico y comprensible (Kaufmann y Gil Aluja, 1993). Este método permite tomar en consideración aspectos no siempre cuantificables de la realidad económica, como los políticos, los sociales, los institucionales, los geográficos, los históricos, entre otros. Así, los expertos utilizan la escala endecadaria y tienen la libertad de expresar sus opiniones, mediante un intervalo de confianza comprendido entre 0 y 1. 
En este sentido, los expertos proporcionan su opinión mediante la progresión del falso al verdadero en la escala endecadaria (Quesada, 2010). La referida escala propia de la lógica difusa, se identifica de la siguiente manera:

\begin{tabular}{|c|c|c|c|c|c|c|c|c|c|c|}
\hline 0 & 0.1 & 0.2 & 0.3 & 0.4 & 0.5 & 0.6 & $0.7 \mid$ & 0.8 & 0.9 & 1 \\
\hline $\begin{array}{l}\circ \\
\frac{0}{\pi} \\
\text { 山 }\end{array}$ & 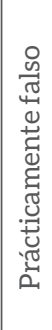 & 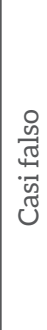 & 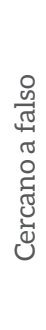 & 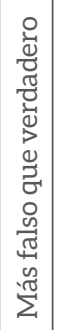 & 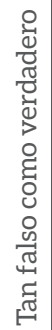 & 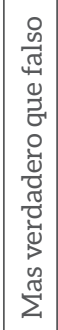 & 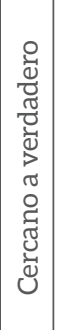 & 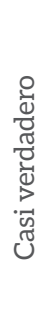 & 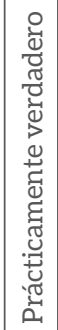 & 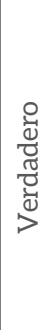 \\
\hline
\end{tabular}

Con la opinión expresada de los expertos en función de los valores de la escala endecadaria, se procede a la construcción del expertizaje de la siguiente manera (Hurtado y Tinto, 2009):

1. Se obtiene la opinión del conjunto de expertos en función del tema seleccionado.

2. Se construye la frecuencia de las opiniones. La opinión de los expertos es reflejada en la escala semántica de acuerdo a los valores que representan para los márgenes derecho e izquierdo respectivamente.

3. Se normaliza la serie. Consiste en dividir los datos entre el número de expertos.

4. Se acumula la frecuencia relativa, con lo que se obtiene el expertón. Consiste en sumar los valores de abajo hacia arriba hasta totalizar la unidad.

5. Se calcula la media aritmética de los datos. Se suman los datos sin tomar en cuenta los valores ubicados en la casilla de cero y se divide el cociente entre diez. De esta manera se obtiene la media aritmética de las opiniones de los expertos.
A través de esta técnica se da cabida a las opiniones reflejadas en patrones subjetivos (casi, bastante, cerca, entre otros) que de igual forma permiten conseguir resultados comprensibles y entendibles a la razón humana.

A manera de ejemplo para ilustrar el procedimiento del expertizaje, se plantea definir las contribuciones o colaboraciones de padres y representantes en los colegios para el próximo período escolar, se pregunta a los entendidos en la materia su opinión acerca de un valor mínimo (pesimista) y un valor máximo (optimista) en que se podría situar la referida colaboración y se obtienen las siguientes opiniones que se refieren al precio en bolívares de las contribuciones por estudiante que deben realizar los padres y representantes (ver cuadro 1).

\section{Cuadro 1. Contribución de los padres y representante por estudiantes (en Bolívares)}

\begin{tabular}{|c|c|c|}
\hline 1 & 500 & 750 \\
\hline 2 & 650 & 900 \\
\hline 3 & 800 & 970 \\
\hline 4 & 750 & 1000 \\
\hline 5 & 600 & 900 \\
\hline 6 & 750 & 950 \\
\hline 7 & 670 & 820 \\
\hline 8 & 800 & 1000 \\
\hline 9 & 700 & 950 \\
\hline
\end{tabular}

Fuente: Elaboración propia.

A partir de la información obtenida por los expertos, se selecciona el menor y mayor valor de este grupo y se consigue así la siguiente banda: (500; 1000), lo 
que representa que la contribución por estudiante que deben realizar los padres para el próximo período escolar podrá estar entre 500 y 1000 bolívares.

Posteriormente se siguen los demás pasos de la construcción del expertizaje, en primer lugar, se consulta la opinión de los expertos en función de la banda obtenida entre el valor pesimista y optimista, para lo cual se utiliza la escala endecadaria propia de la lógica difusa. En segundo lugar, se construye la frecuencia de las opiniones, en el ejemplo planteado el número de expertos es 9. En tercer lugar, se normaliza la serie, luego se obtiene la frecuencia relativa que permite conseguir el expertón y por último se calcula la media aritmética de los datos.

Una vez que se obtiene la media aritmética de las opiniones de los expertos se concluye que estas se inclinan en una proporción de 0.82 hacia el escenario de que la contribución se ubique en 1000 bolívares por estudiante. Dándole cabida a opiniones con patrones subjetivos que permiten conseguir resultados comprensibles y entendibles al racionamiento humano.

\section{Contraexpertizaje}

Cuando se quiere aumentar la calidad de los resultados, reduciendo la entropía e incertidumbre de los mismos, se acude al método del contraexpertizaje que según Kaufmann y Gil (1993), representa una herramienta de la lógica difusa que permite tomar una nueva opinión de expertos acerca de un tema que ya ha sido evaluado por expertos, es decir, toma una nueva opinión de expertos acerca de un tema ya evaluado por expertos; representa un medio para reducir la entropía e incertidumbre de resultados previamente obtenidos, aumenta su calidad y da cabida a nuevas opiniones de expertos del tema estudiado. En otras palabras, el contraexpertizaje no es más que la opinión generada desde la perspectiva de varios expertos con respecto a un determinado intervalo proveniente del expertizaje, cuya finalidad es reducir su rango o banda para hacerlo menos incierto.

Siguiendo con el ejemplo planteado anteriormente, se busca con esta técnica, reducir la banda propuesta de la

Cuadro 2. Construcción del expertizaje

\begin{tabular}{|c|c|c|c|c|c|c|c|c|c|c|c|}
\hline & & & 0 & 0 & 0 & 0 & 0 & 1 & 1 & 1 & 1 \\
\hline & & & 0.1 & 0 & 0 & 0.00 & 0.00 & 1.00 & 1.00 & 1.00 & 1.00 \\
\hline Experto 1 & 0.5 & 0.7 & 0.2 & 0 & 0 & 0.00 & 0.00 & 1.00 & 1.00 & 1.00 & 1.00 \\
\hline Experto 2 & 0.4 & 0.6 & 0.3 & 0 & 0 & 0.00 & 0.00 & 1.00 & 1.00 & 1.00 & 1.00 \\
\hline Experto 3 & 0.9 & 1 & 0.4 & 1 & 0 & 0.11 & 0.00 & 1.00 & 1.00 & 1.00 & 1.00 \\
\hline Experto 4 & 0.8 & 0.9 & 0.5 & 3 & 0 & 0.33 & 0.00 & 0.89 & 1.00 & 0.89 & 1.00 \\
\hline Experto 5 & 0.7 & 0.8 & 0.6 & 1 & 1 & 0.11 & 0.11 & 0.56 & 1.00 & 0.56 & 1.00 \\
\hline Experto 6 & 0.5 & 0.9 & 0.7 & 2 & 2 & 0.22 & 0.22 & 0.44 & 0.89 & 0.44 & 0.89 \\
\hline Experto 7 & 0.7 & 1 & 0.8 & 1 & 2 & 0.11 & 0.22 & 0.22 & 0.67 & 0.22 & 0.67 \\
\hline Experto 8 & 0.6 & 0.8 & 0.9 & 1 & 2 & 0.11 & 0.22 & 0.11 & 0.44 & 0.11 & 0.44 \\
\hline \multirow[t]{3}{*}{ Experto 9} & 0.5 & 0.7 & 1 & 0 & 2 & 0.00 & 0.22 & 0.00 & 0.22 & 0.00 & 0.22 \\
\hline & & & & & & & & & & 0.62 & 0.82 \\
\hline & \multicolumn{2}{|c|}{$\begin{array}{l}\text { Opinión de } \\
\text { Expertos }\end{array}$} & & \multicolumn{2}{|c|}{$\begin{array}{c}\text { Frecuencia de } \\
\text { las opiniones }\end{array}$} & \multicolumn{2}{|c|}{$\begin{array}{c}\text { Serie } \\
\text { Normalizada }\end{array}$} & \multicolumn{2}{|c|}{$\begin{array}{l}\text { Frecuencias } \\
\text { Acumuladas }\end{array}$} & \multicolumn{2}{|c|}{$\begin{array}{c}\text { Media } \\
\text { Aritmética } \\
\text { e los datos }\end{array}$} \\
\hline
\end{tabular}

Fuente: Elaboración propia. 
contribución de los padres y representante en los colegios a partir de una nueva opinión de expertos quienes opinaran sobre la banda $(500 ; 1000)$ bolívares utilizando la siguiente escala semántica:

\begin{tabular}{|r|l|}
\hline 0 & El valor de 500 es el correcto \\
\hline 0.1 & Prácticamente 500 \\
\hline 0.2 & Casi 500 \\
\hline 0.3 & Cercano a 500 \\
\hline 0.4 & Más cerca de 500 que de 1.000 \\
\hline 0.5 & Tan cerca de 500 como de 1.000 \\
\hline 0.6 & Más cerca de 1.000 que de 500 \\
\hline 0.7 & Cercano a 1.000 \\
\hline 0.8 & Casi 1.000 \\
\hline 0.9 & Prácticamente 1.000 \\
\hline 1 & El valor de 1.000 es el correcto \\
\hline
\end{tabular}

Se consulta a los nuevos expertos quienes de acuerdo a dicha escala dicen:

\section{Cuadro 3. Opinión de nuevos experto}

\begin{tabular}{|c|c|}
\hline 1 & $0.5 ; 0.7$ \\
\hline 2 & $0 ; 1$ \\
\hline 3 & $0.2 ; 1$ \\
\hline 4 & $0.4 ; 0.8$ \\
\hline 5 & $0.6 ; 0.9$ \\
\hline
\end{tabular}

Fuente: Elaboración propia.
A partir de esto y de acuerdo a Hurtado y Tinto (2009) el procedimiento para construir el contraexpertizaje es el siguiente: 1) se vacía la información de los nuevos expertos para descubrir la frecuencia de estas opiniones (número de expertos 5); 2) se normalizan los datos, dividiendo las frecuencias entre el número de expertos; 3) se acumula la frecuencia relativa; 4) se evalúa la nueva opinión de los expertos a través de la siguiente identidad matemática:

$$
A(+)\left[A^{*}-A\right](x) \text { Expertón }
$$

En donde:

$[500,1.000]=\left[\mathrm{A}, \mathrm{A}^{*}\right]$

Expertón: valores que se obtienen al acumular la frecuencia relativa.

Sustituyendo en la fórmula:

$500+(1.000-500)$ x Expertón

500 (500) x Expertón

Por último, Se calcula la media aritmética de los datos. Se suman los datos sin tomar en cuenta los valores ubicados en la casilla de cero y se divide el cociente entre diez.

\section{Cuadro 4. Construcción del contraexpertizaje}

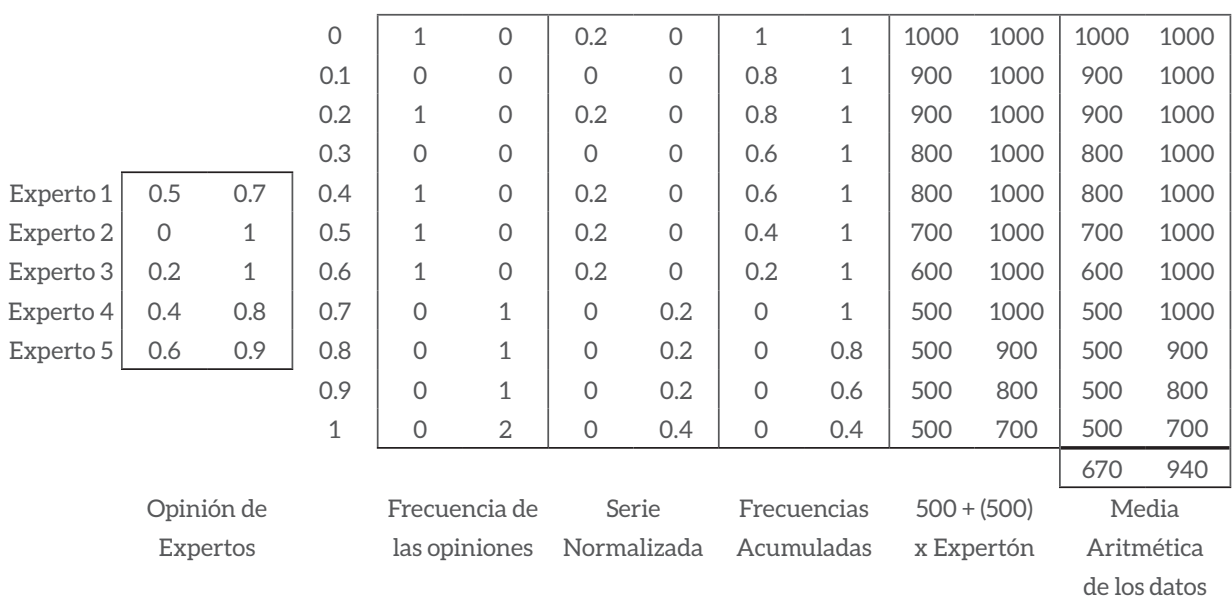

Fuente: Elaboración propia. 
De acuerdo con la opinión de los expertos, se obtiene que la contribución - colaboración de los padres y representante esté entre Bs. 670 y Bs. 940 por estudiante para comienzos del próximo periodo escolar. De esta forma se ha disminuido la incertidumbre que presenta la propuesta inicla, por lo que se gana precisión al reducir la entropía presente en los resultados y se evita la utilización y presentación de información incorrecta. Es por ello que este conjunto de herramientas representa las técnicas de lógica difusa que permitirán manejar el conjunto de variables relacionadas a la eficiencia de los programas sociales en educación.

\section{Ratios inciertos}

Este método permite estudiar hechos pasados y presentes para realizar previsiones futuras de una variable o premisa examinada, además, permite realizar comparaciones entre situaciones. El cálculo de ratios inciertos requiere de la consideración de ciertas normas para la realización de comparaciones homogéneas (Hurtado y Tinto, 2009). Los ratios inciertos inducen la necesidad de incluir múltiples aspectos de una situación o hecho cuyo fin sea obtener una visión global.

Para Hurtado, Tinto y Zerpa (2011) la metodología de ratios plantea relaciones de cocientes en las cuales el numerador y denominador semueven en función de una misma variable, lo que se complementa con el manejo de intervalos de confianza como medio para percibir las situaciones de incertidumbre. Para ello, es necesario que los criterios de valoración sean construidos por los ratios de una fuente confiable procedente de organizaciones gubernamentales, académicas $\mathrm{de}$ investigación aún en épocas diferentes, ya que los procedimientos llevados a cabo con dichas fuentes en diferentes momentos arrojan una comparación entre datos. La característica particular de esta herramienta es el estudio comparativo, dado que los ratios considerados aisladamente presentan una utilidad relativa (Gil, 1999).

\section{Construcción del modelo}

Se construyó un modelo, que describe de manera integral la eficiencia que han tenido las misiones sociales en educación en la zona de estudio, esto se logró mediante la evaluación de la eficiencia de las políticas sociales a través de los enfoques cuantitativos y cualitativos. La utilización de la lógica difusa para la valoración de las variables e indicadores permitió la evaluación de la eficiencia de las referidas misiones sociales desde un umbral o intervalo de confianza.

\section{Selección de componentes e indicadores}

Las restricciones expuestas por la multiplicidad de métodos existentes para evaluar las políticas sociales han llevado a la selección del área de estudio y a la construcción de indicadores que integren una diversidad de variables que logren superar dichas limitaciones. Con base a esto surge la necesidad de describir los requerimientos básicos que deben cumplirse para la selección adecuada de dichas variables. Un indicador es la evidencia de la obtención del resultado, del cambio, del logro que se busca alcanzar con un programa o un proyecto; el indicador es un criterio que permite evaluar ese cambio. Así mismo, los indicadores representan una importante herramienta para la toma de decisiones ya que resultan fundamentales para 
evaluar y proyectar tendencias de la situación de una región en lo referente a cuestiones económicas y sociales, además que permiten valorar el cumplimiento de las metas y objetivos fijados en las políticas de gobierno (López y Gentile, 2008).

En función de lo antes expuesto y de acuerdo con lo planteado por Picado (1997) los requerimientos que debe tener un indicador son: 1) ser confiable y 2) ser válidos. La confiablidad se refiere a la precisión o consistencia en la medición; un indicador será confiable en la medida en que aplicado en las mismas condiciones produzca los mismo resultados. La validez se refiere a la capacidad en que la medida seleccionada empíricamente refleje en forma adecuada el significado real de lo que se busca medir.

Dentro de este contexto, se han seleccionado diferentes indicadores para analizarlos con precisión, teniendo en cuenta aspectos como el potencial de aplicación de políticas públicas, la pertinencia en el monitoreo y control de la realidad en estudio, la determinación de dimensiones subjetivas y objetivas, y la adaptabilidad a la realidad del Área Metropolitana del Estado Mérida. Así, se determinó el siguiente grupo de componentes para explicar la eficiencia de las misiones sociales en educación: 1) contexto, 2) recursos, 3) proceso y 4) avances. Se contempla una perspectiva subjetiva de los datos, así como otra objetiva. Con base en estos criterios se seleccionaron lo siguientes indicadores que permiten identificar, comparar $y$ calificar las características de la política social de educación implementada por el gobierno.

\section{Cuadro 5. Indicadores de eficiencia por componente.}

\begin{tabular}{|l|l|}
\hline Componente & \multicolumn{1}{|c|}{ Indicadores } \\
\hline Contexto & $\begin{array}{l}\text { Índice de pobreza } \\
\text { Coeficiente de Gini } \\
\text { Percepción del gasto en } \\
\text { educación }\end{array}$ \\
\hline Recursos & $\begin{array}{l}\text { Número de docentes } \\
\text { Número de edificios e } \\
\text { instalaciones } \\
\text { Asignación presupuestaria }\end{array}$ \\
\hline Proceso & $\begin{array}{l}\text { Número de estudiante por } \\
\text { aula } \\
\text { Percepción del proceso } \\
\text { docente }\end{array}$ \\
\hline Avances & $\begin{array}{l}\text { Cobertura (número de } \\
\text { inscritos) } \\
\text { Impacto (cociente entre el } \\
\text { número de egresado y el } \\
\text { número total de inscrito) } \\
\text { Calidad (satisfacción de los } \\
\text { beneficiarios) }\end{array}$ \\
\hline
\end{tabular}

Fuente: Elaboración propia

\section{Normalización de los indicadores}

Se seleccionó el método de ratios inciertos para la determinación del nuevo modelo de eficiencia de las misiones de educación en el Área Metropolitana del Estado Mérida a partir de las ventajas de la lógica difusa en el proceso de normalización y estandarización de los indicadores cuantitativos. Para ello se integraron a los indicadores que describen el ámbito objetivo del mejor uso de los recursos escasos, todos aquellos indicadores que explican el ámbito subjetivo del término de eficiencia. La información de estos últimos fue homogenizada con la utilización de la técnica de expertizaje, que como herramienta permitió convertir la información subjetiva, vaga e incierta a la misma escala referida para las variables objetivas. De esta forma, se establecieron los nuevos indicadores subjetivos por componentes, se normalizaron los mencionados indicadores, se agregaron a 
los indicadores objetivos y se determinó el nuevo modelo de eficiencia de las misiones educativas a partir de un umbral de confianza, con el cual se explica la eficiencia de las misiones educativas desde un enfoque multidimensional, incorporando así, las mediciones objetivas de eficiencia y, por otra parte, la valoración desde la perspectiva propia de los principales beneficiarios de dichos programas, de esta manera se ilustra un análisis de la eficiencia de las misiones sociales en educación evitando realizarse solo por medio de la información objetiva, sino que se incluye la información que suministra el individuo sobre su participación.

\section{Información estadística}

Las fuentes de información utilizadas para el cálculo de los indicadores han sido tanto de orden primario como secundario. De esta manera, para identificar los datos necesarios para precisar los indicadores que describen las variables cualitativas fue necesario el diseño y aplicación de la Encuesta Final de Eficiencia Educación, que como elemento estadístico permitió integrar los principios de lógica difusa en la recolección de la información, y distinguir aspectos claves para explicar la eficiencia de las misiones educativas en el área objeto de estudio. La aplicación de dicha encuesta fue destinada a los participantes de cada uno de los programas educativos y se realizó para tres años consecutivos (2013, 2014, 2015). Se definió una muestra representativa de la población de 330 unidades de estudio, siendo el objeto de observación y estudio los beneficiarios de las misiones educativas del Área Metropolitana del Estado Mérida. El tamaño de la muestra y la distribución de la misma, se correspondió con las pautas utilizadas en la IV Encuesta Nacional de Presupuestos Familiares (ENPF) que durante 2008 y 2009 fue aplicada en la región, de manera conjunta, por la Universidad de Los Andes (ULA), el Banco Central de Venezuela (BCV) y el Instituto Nacional de Estadística (INE).

Además de la aplicación de la mencionada encuesta, se realizó una profunda revisión de distintas fuentes secundarias de información, con el fin de identificar cada uno de los indicadores cuantitativos del modelo, las principales fuentes secundarias fueron: Ministerio del Poder Popular para Educación Universitaria, Ciencia y Tecnología; Ministerio del Poder Popular para la Educación; Ministerio del Poder Popular de Petróleo y Minería, y se revisaron las memorias y cuentas de dichos ministerios para obtener los datos de asignación presupuestaria, datos sobre número de inscritos y egresados, así mismo, resultó necesario la utilización de fuentes bibliográficas tales como libros, artículos, artículos de revistas, trabajos de investigación, base de datos digitales, entre otras, las cuales permitieron la identificación de los indicadores antes descritos.

\section{Resultados}

Partiendo de la idea de que el nivel de eficiencia que han tenido las misiones sociales en educación y su efecto en la sociedad venezolana se explica desde un enfoque integrador de descriptores objetivos y subjetivos, se determinó el nuevo modelo de eficiencia de las misiones educativas en el Área Metropolitana del estado Mérida. El mismo se estimó a partir del uso de las herramientas de lógica difusa para el manejo de las variables cuantitativas 
y cualitativas seleccionadas con el propósito de explicar cada indicador de eficiencia de la misión entre 2013 y 2015, ello debido a que la información subjetiva de fuente primaria solo se pudo recoger para esos años.

De esta manera, se revisó la evolución de cada uno de los indicadores previamente definidos: contexto, recursos, proceso, avances; en principio de forma individual y luego de manera agregada para conseguir la nueva medida de eficiencia de la política social en estudio. Esta medida se construyó a partir de la agregación de variables por el método de ponderación simple, pero haciendo énfasis en la necesidad de utilizar los operadores de números borrosos para agregar los intervalos de confianza que explican cada indicador utilizado en el estudio.

Los resultados obtenidos están comprendidos por valores que van del 0 al 1. Se identifica con cero a la peor situación, es decir, ineficiencia, y con uno, la mejor situación: eficiencia. Con dicha idea, se utilizó la escala endecadaria que se muestra a continuación para realizar el análisis de los resultados.

0 Ineficiente

0.1 prácticamente ineficiente

0.2 casi ineficiente

0.3 cercano a ineficiente

0.4 más ineficiente que eficiente

0.5 Tan ineficiente como eficiente

0.6 más eficiente que ineficiente

0.7 cercano a eficiente

0.8 casi eficiente

0.9 prácticamente eficiente

1 Eficiente

\section{Contexto}

Para el análisis del componente contexto se utilizaron las variables: a) índice de pobreza (IP), b) coeficiente de Gini (CG) y c) percepción del gasto en educación de los beneficiarios de las misiones educativas en el Área Metropolitana del estado Mérida (PGE). La selección de estos indicadores permitió identificar los aspectos socioeconómicos de la población objeto de estudio.

En el caso de las dos primeras variables de carácter objetivo, estas se estandarizaron utilizando el método de ratios inciertos, mientras que la tercera variable de carácter subjetivo se normalizó mediante el cálculo del expertizaje. Para este último procedimiento se utilizó la información primaria disponible de la Encuesta Final de Eficiencia Educación aplicada a los participantes seleccionados de las distintas zonas del Área Metropolitana del estado Mérida.

La técnica del expertizaje se desarrolló de la siguiente manera:

a) Se consultó la opinión de los usuarios del Área Metropolitana del Estado Mérida acerca del gasto realizado en educación.

b) Se plantearon las siguientes alternativas que explican el gasto en educación realizado por los habitantes de la zona en estudio. Mientras menor sean los recursos que deben destinar las personas a su educación, mayor será el acceso de la población al servicio que prestan los programas sociales educativos. Empleando la escala endecadaria de la lógica difusa se reveló la percepción de los usuarios sobre el gasto que realizan en educación, obteniéndose las siguientes opiniones: 
¿Cuánto gasta mensualmente en Educación?

\begin{tabular}{|c|c|c|c|c|c|c|c|c|c|c|c|}
\hline & 0 & 0.1 & 0.2 & .0 .3 & 0.4 & 0.5 & 0.6 & 0.7 & 0.8 & 0.9 & 1 \\
\hline Menor a Bs. 1.500 & 66 & 15 & 19 & 16 & 8 & 4 & 8 & 5 & 6 & 7 & 176 \\
\hline $\begin{array}{c}\text { Entre Bs. } 1.500 \text { y menos de } \\
\text { Bs } 3.000\end{array}$ & 294 & 2 & 3 & 1 & 1 & 2 & 3 & 8 & 8 & 5 & 3 \\
\hline $\begin{array}{c}\text { Entre Bs. 3.000 y menos de } \\
\text { Bs. } 4.500\end{array}$ & 327 & 0 & 0 & 0 & 0 & 1 & 0 & 0 & 0 & 0 & 2 \\
\hline $\begin{array}{c}\text { Entre Bs. } 4.500 \text { y menos de } \\
\text { Bs. } 6.000\end{array}$ & 330 & 0 & 0 & 0 & 0 & 0 & 0 & 0 & 0 & 0 & 0 \\
\hline Mayor a Bs 6.000 & 330 & 0 & 0 & 0 & 0 & 0 & 0 & 0 & 0 & 0 & 0 \\
\hline
\end{tabular}

Fuente: Encuesta Final de Eficiencia Educación. Elaboración propia.

c) Se elaboró el expertizaje a partir de la utilización de la alternativa Entre Bs 1.500 y menos de Bs 3.000 como medio para describir el grado de menor accesibilidad (Li), y el planteamiento menor a Bs 1.500 como instrumento para explicar mayor accesibilidad (Ls). Con la utilización de la información descrita en ambas opciones, como intervalo de confianza, fue posible evaluar la percepción del gasto en educación. d) Se consiguió el siguiente resultado, explicado en el cuadro 6.

En este sentido, mediante el rango obtenido se concluyó que los participantes de las misiones educativas en el Área Metropolitana del estado Mérida evaluaron su nivel de gasto en educación a través de un umbral que va desde 0.072 , poca accesibilidad, hasta 0.638 considerándolo más cercano a accesible, esto explica que la utilización de las

\section{Cuadro 6. Percepción del gasto en educación (2013)}

\begin{tabular}{|c|c|c|c|c|c|c|c|c|}
\hline & \multicolumn{2}{|c|}{ Frecuencia de opiniones } & \multicolumn{2}{|c|}{ Serie Normalizada } & \multicolumn{2}{|c|}{ Frecuencia Acumulada } & \multicolumn{2}{|c|}{ Media Aritmética de los datos } \\
\hline & $\begin{array}{c}\mathrm{N}^{\circ} \mathrm{de} \\
\text { Respuestas } \\
\text { Li }\end{array}$ & $\begin{array}{c}\mathrm{N}^{\circ} \mathrm{de} \\
\text { Respuestas } \\
\text { Ls }\end{array}$ & $\begin{array}{c}\mathrm{N}^{\circ} \mathrm{de} \\
\text { Respuestas/ } \\
\mathrm{N}^{\circ} \text { de } \\
\text { Expertos }\end{array}$ & $\begin{array}{c}\mathrm{N}^{\circ} \mathrm{de} \\
\text { Respuestas/ } \\
\mathrm{N}^{\circ} \text { de } \\
\text { Expertos } \\
\end{array}$ & $\begin{array}{c}\text { Suma } \\
\text { ascendente } \\
\text { de valores }\end{array}$ & $\begin{array}{c}\text { Suma } \\
\text { ascendente } \\
\text { de valores }\end{array}$ & $\begin{array}{c}* \text { Suma de los } \\
\text { datos } / 10\end{array}$ & $\begin{array}{c}\text { * Suma de los } \\
\text { datos } / 10\end{array}$ \\
\hline 0 & \multicolumn{2}{|l|}{294} & \multicolumn{2}{|l|}{0.891} & \multicolumn{2}{|l|}{1} & \multicolumn{2}{|l|}{1} \\
\hline 0.1 & \multicolumn{2}{|r|}{15} & \multicolumn{2}{|l|}{0.006} & \multicolumn{2}{|l|}{0.109} & 0.109 & 0.800 \\
\hline 0.2 & \multicolumn{2}{|r|}{19} & \multicolumn{2}{|l|}{0.009} & \multicolumn{2}{|l|}{0.103} & 0.103 & 0.755 \\
\hline 0.3 & \multicolumn{2}{|l|}{1} & 0.003 & 0.052 & 0.094 & 0.697 & 0.094 & 0.697 \\
\hline 0.4 & \multicolumn{2}{|l|}{1} & 0.003 & 0.024 & 0.091 & 0.648 & 0.091 & 0.648 \\
\hline 0.5 & \multicolumn{2}{|l|}{2} & 0.006 & 0.012 & 0.088 & 0.624 & 0.088 & 0.624 \\
\hline 0.6 & \multicolumn{2}{|l|}{3} & 0.009 & 0.021 & 0.082 & 0.612 & 0.082 & 0.612 \\
\hline 0.7 & \multicolumn{2}{|l|}{8} & 0.024 & 0,015 & 0.073 & 0.588 & 0.073 & 0.588 \\
\hline 0.8 & \multicolumn{2}{|l|}{8} & 0.024 & 0.018 & 0.048 & 0.573 & 0.048 & 0.573 \\
\hline 0.9 & \multicolumn{2}{|l|}{5} & 0.015 & 0.021 & 0.024 & 0.555 & 0.024 & 0.555 \\
\hline 1 & \multicolumn{2}{|r|}{176} & 0.009 & 0.533 & 0.009 & 0.533 & 0.009 & 0.533 \\
\hline & & & & & & & 0.072 & 0.638 \\
\hline
\end{tabular}

Fuente: Cálculos propios

* Se suman todos los datos sin tomar en consideración los valores ubicados en la casilla de cero. 
misiones les permitió integrarse al sistema educativo durante el 2013. Para 2014 las personas evaluaron el gasto en educación mediante la calificación de [0.221, 0.561], lo que significa que las personas percibieron un grado de accesibilidad que va desde un nivel casi bajo a un nivel entre bajo y alto de accesibilidad. Para 2015 referido umbral cambio hasta [0.072, 0.640], lo que muestra que la percepción en cuanto al gasto en educación llegó a estar entre niveles de prácticamente nula accesibilidad y más accesible que inaccesible, lo que demuestra el impacto que la coyuntura de la economía nacional (alta inflación, escasez y desabastecimiento) ha provocado en la disponibilidad de estas políticas sociales y en la disposición de las personas para considerarlas como alternativa de educación formal.

En relación con los indicadores objetivos que forman parten del componente contexto, Índice de Pobreza (IP) y Coeficiente de Gini (CG), su normalización se hizo mediante ratios inciertos, dichos parámetros objetivos alcanzaron para el 2013 calificaciones de 0.128 y 0.591 respectivamente. Para 2014 ambos parámetros llegaron a niveles de 0.126 y 0.620 , debido al incremento del nivel de desigualdad en la distribución de la renta en el Área Metropolitana del estado Mérida. Finalmente para 2015 dichas variables terminaron con valores de 0.124 y 0.619 , tal como se observa en la tabla 1 , mostrando una tendencia a la reducción de los valores descritos.

Luego de obtener los resultados de los indicadores de manera individual se desarrolló el cálculo del aporte del componente contexto al índice de eficiencia de las misiones educativas del área de estudio. En este sentido, se utilizaron los operadores de matemática borrosa para realizar la agregación como promedio simple de los datos de cada indicador, obteniéndose para 2013 el resultado que se presenta a continuación.

$$
\begin{gathered}
\stackrel{I}{\sim}_{C}=\frac{I P+C G+P G E}{3} \\
\stackrel{\sim}{C}_{C}=\frac{[0.128,0.128]+[0.591,0.591]+[0.072,0.638]}{3} \\
\sim_{C}=[0.264,0.452]
\end{gathered}
$$

El dato obtenido explica que la eficiencia del componente contexto en el Área Metropolitana del Estado Mérida, según la escala endecadaria propia de la lógica difusa, estuvo durante 2013 en un rango entre casi ineficiente y cercano a ineficiente, descrita por la calificación [0.264, 0.452]. De esta manera se logra conjuntar análisis objetivo y subjetivo para explicar los aspectos socioeconómicos de la población considerada para estudiar la eficiencia de las misiones educativas como política social. Es necesario recalcar que para el cálculo de todas las variables cuantitativas se utilizó el dato real obtenido por las instituciones encargadas de compilar el mismo mediante procedimientos estadísticos, donde se hace caer la entropía presente en la realidad desde primer instante, por consiguiente, para dicha información se consideró un mismo valor en el límite inferior y superior de los umbrales, es decir, parámetros como el siguiente: [0.5, 0.5]. Lo anteriormente expuesto no limita la agregación de los umbrales de las variables cuantitativas y cualitativas a través de operadores de matemática borrosa.

Para terminar el análisis de la evolución del componente contexto durante el periodo en estudio se realizó igual procedimiento para los años 2014 y 2015. Al respecto, de los referidos cálculos se obtuvo para el componente contexto en 2014 el siguiente rango [0.322, 0.436], lo que quiere decir, que 
alcanzó un intervalo entre más ineficiente que eficiente. En lo que se refiere a 2015, se colocó en un umbral entre casi ineficiente y más ineficiente que eficiente, que está representado por la banda [0.272, 0461], tal como se identifica en la tabla 1.

\section{Tabla 1. Evolución del componente contexto y sus indicadores}

\begin{tabular}{|l|c|c|c|c|c|c|}
\hline \multicolumn{1}{|c|}{ Años } & \multicolumn{2}{|c|}{2013} & \multicolumn{2}{c|}{2014} & \multicolumn{2}{c|}{2015} \\
\hline $\begin{array}{l}\text { Indicador de } \\
\text { Contexto }\end{array}$ & 0.264 & 0.452 & 0.322 & 0.436 & 0.272 & 0.461 \\
\hline $\begin{array}{l}\text { Índice de } \\
\text { Pobreza }\end{array}$ & 0.128 & 0.128 & 0.126 & 0.126 & 0.124 & 0.124 \\
\hline $\begin{array}{l}\text { Coeficiente } \\
\text { de Gini }\end{array}$ & 0.591 & 0.591 & 0.620 & 0.620 & 0.619 & 0.619 \\
\hline $\begin{array}{l}\text { Percepción } \\
\text { del gasto en } \\
\text { educación }\end{array}$ & 0.072 & 0.638 & 0.221 & 0.561 & 0.072 & 0.640 \\
\hline
\end{tabular}

Fuente: Elaboración propia

\section{Recursos}

Para el caso del componente recursos, se procedió a la identificación de cada uno de los indicadores para el periodo en estudio. Se utilizaron las variables: a) Número de docentes (D); b) Número de edificios e instalaciones (EI) y c) Asignación presupuestaria (AP), con el fin de evaluar el estado y provisión de bienes y servicios, así como el uso de los recursos necesarios para el logro de los objetivos planteados en las misiones educativas. El parámetro número de docentes explica la cantidad de facilitadores con que cuenta cada misión educativa, en este sentido, cada misión según su objetivo cuenta con una cantidad diferente de docentes necesarios para atender a los participantes. Por su parte el indicador número de edificios e instalaciones explica si los espacios acondicionados para atender a los usuarios son suficientes y adecuados para tal propósito. Por último el indicador asignación presupuestaria explica la eficiencia en el uso de los recursos para el cumplimiento de las metas propuestas por cada misión en el periodo en estudio.
Para la normalización de las variables se utilizó el método de ratios inciertos, y se tomaron en cuenta los datos de cada una de las misiones educativas (Misión Robinson I, II; Misión Ribas y Misión Sucre), las cuales se agregaron haciendo uso de las operaciones de matemática borrosa de tal forma de obtener cada uno de los parámetros que componen la dimensión recursos. De esta manera para el periodo 2013 el parámetro número de docentes se explicó como prácticamente ineficiente corroborado por la calificación [0.155, 0.155], lo que implica un déficit en la cantidad de facilitadores que se requieren para atender de manera adecuada a los participantes de las misiones educativas. Esta tendencia se mantuvo para los años siguientes del periodo en estudio. En 2014 el umbral se ubicó hasta [0.151, 0.151], con lo cual se describió una situación de prácticamente ineficiente, y con relación a 2015 se acortó el intervalo explicado mediante el rango [0.139, 0.139], ver tabla 2.

De igual forma se utilizó el método de ratios inciertos para los indicadores número de edificios e instalaciones, y asignación presupuestaria. En cuanto al parámetro número de edificios e instalaciones para 2013 se describió como casi ineficiente explicado por un umbral igual a [0.293, 0.293], tendencia que se mantuvo para los años siguientes, ya que para 2014 se obtuvo el mismo umbral, debido a que el número de planteles sedes no se modificaron en el Área Metropolitana del Estado Mérida; mientras que en 2015 se continúa describiendo los resultados de esta variable como casi ineficiente, pero esta vez corroborado por un nivel de [0.262, 0.262], alcanzado por la reducción del número de establecimientos disponibles para la misión en el área de estudio. En relación con la variable asignación presupuestaria, esta permitió 
identificar la baja eficiencia en el uso de los recursos por parte de cada una de las misiones educativas, evidenciando que no cubren de manera significativas todas las actividades que permitan cumplir con los objetivos y metas de mencionados programas, para 2013 se obtuvo como resultado un umbral de[0.030,0.030] loque describe una situación de ineficiencia en el uso de los recursos financieros necesarios para el funcionamientos de los distintitos programas educativos en la región de estudio, así mismo para 2014 la tendencia se conservó ratificado con la calificación de [0.031, 0.031], y para 2015 con un nivel de [0.033, 0.033], esto se explica porque la asignación presupuestaria de estos programas dependen totalmente de los entes públicos a los cuales están adscritos.

Al tener identificados cada uno de los indicadores fue posible la agregación con la intención de conocer la medida de la dimensión recursos. Una vez realizado el procedimiento de agregación, mediante el uso de operaciones de matemática difusa, se obtuvieron los siguientes resultados:

$$
\begin{gathered}
\sim_{R}^{I}=\frac{D+E I+A P}{3} \\
\stackrel{\sim}{R}_{R}=\frac{[0.1559,0.155]+[0.293,0.293]+[0.030,0.630]}{3} \\
\underset{\sim}{I}=[0.159,0.159]
\end{gathered}
$$

De estos se deducen que la eficiencia del componente recursos de las misiones educativas en el Área Metropolitana del Estado Mérida se ubicó durante 2013 en un umbral de [0.159, 0.159] lo cual se describe como prácticamente ineficiente, este resultado se debe a una insuficiencia en la dotación de los recursos materiales y humanos indispensables para su debido funcionamiento, así como el espacio físico donde se llevan a cabo los programas no cuenta con las condiciones necesarias para instrumentarlos. Así como, la dependencia del país de la renta petrolera para subsanar los problemas sociales que atraviesa la sociedad venezolana, estrategia que se ha visto afectada por la disminución del precio de los últimos años. Mientras que para 2014 se colocó de igual forma en un intervalo de prácticamente ineficiente que se explica por las calificaciones [0.158, 0.158], para finalizar con la evolución del indicador de recursos en 2015 que alcanzó un nivel de [0.145,0.145], estrechándose cada vez más el umbral explicado en la tabla 2 .

\begin{tabular}{|c|c|c|c|}
\hline Años & 2013 & 2014 & 2015 \\
\hline $\begin{array}{l}\text { Indicador de } \\
\text { Recursos }\end{array}$ & 0.1590 .159 & 0.1580 .158 & 0.1450 .145 \\
\hline $\begin{array}{l}\mathrm{N}^{\circ} \text { de } \\
\text { Docentes }\end{array}$ & 0.1550 .155 & $0.151 \quad 0.151$ & 0.1390 .139 \\
\hline $\begin{array}{l}\mathrm{N}^{\circ} \text { de } \\
\text { edificios e } \\
\text { instalaciones }\end{array}$ & 0.2930 .293 & 0.2930 .293 & 0.2620 .262 \\
\hline $\begin{array}{l}\text { Asignación } \\
\text { Presupues- } \\
\text { taria }\end{array}$ & 0.0300 .030 & 0.0310 .031 & 0.0330 .033 \\
\hline
\end{tabular}

\section{Tabla 2. Evolución del componente recursos y sus indicadores}

Fuente: Elaboración propia

\section{Proceso}

Con respecto a la dimensión proceso, la cual permite describir las características ambientales y organizacionales de los sistemas educativos, se identificó como indicador de carácter objetivo el número de estudiantes por aula, que se estandarizó a través del método de ratios inciertos. De igual forma se seleccionó como indicador subjetivo la percepción del proceso docente, que se normalizó mediante el cálculo del expertizaje. Para esta última operación se utilizó la información descrita en la Encuesta Final de Eficiencia Educación aplicada a los usuarios de las misiones educativas en el Área Metropolitana del Estado Mérida. 
En este sentido, para el parámetro percepción del proceso docente fue necesario preguntar a los participantes de los distintos programas sociales educativos acerca del método de enseñanza aplicado por los facilitadores en cada una de las referidas misiones. Con dicho propósito se formuló, dentro de las interrogantes incluidas en el cuestionario de la Encuesta Final de Eficiencia Educación la siguiente pregunta ¿Cuál es el método de enseñanza de la misión en la que usted participa?, para la cual se obtuvieron las siguientes opiniones:

\section{¿Cuál es el método de enseñanza de la misión en la que usted participa?}

\begin{tabular}{|l|c|c|c|c|c|c|c|c|c|c|c|}
\hline & 0 & 0.1 & 0.2 & 0.3 & 0.4 & 0.5 & 0.6 & 0.7 & 0.8 & 0.9 & 1 \\
\hline Andragogía & 312 & 0 & 0 & 0 & 0 & 0 & 0 & 0 & 0 & 0 & 18 \\
\hline Tradicional & 222 & 0 & 0 & 0 & 0 & 1 & 1 & 1 & 4 & 12 & 89 \\
\hline Humanista & 264 & 0 & 1 & 0 & 2 & 1 & 0 & 2 & 3 & 5 & 52 \\
\hline $\begin{array}{c}\text { Socialización } \\
\begin{array}{c}\text { Participando } \\
\text { en la } \\
\text { Comunidad }\end{array}\end{array}$ & 276 & 1 & 0 & 0 & 2 & 1 & 1 & 1 & 1 & 2 & 45 \\
\hline
\end{tabular}

Fuente: Encuesta Final de Eficiencia Educación. Elaboración propia.
Se construyó el expertizaje luego de considerar el método tradicional como límite superior y el método humanista como límite inferior. Mediante la utilización de ambas ideas como intervalo de confianza fue posible valuar la condición percepción del proceso docente. Se obtuvo el siguiente resultado, explicado en el cuadro 7:

Con el umbral obtenido se infiere que los beneficiarios de las misiones educativas en el Área Metropolitana del Estado Mérida opinaron en 2013 que el método de enseñanza empleado en las aulas de clase está en un rango que va desde prácticamente humanista a cercano a humanista, representado por el nivel [0.187, 0.328], resultado que corrobora que el método de enseñanza impulsado por estas misiones es concebido como un proceso totalmente humano, de carácter integral, que distingue entre los diferentes ritmos y desarrollos de cada individuo como persona única. Así, en 2014 se obtuvo una calificación de [0.121, 0.300] manteniendo la tendencia de la percepción de los usuarios sobre el proceso docente con intervalo que va

\section{Cuadro 7. Percepción del proceso docente (2013)}

\begin{tabular}{|c|c|c|c|c|c|c|c|c|}
\hline & \multicolumn{2}{|c|}{ Frecuencia de opiniones } & \multicolumn{2}{|c|}{ Serie Normalizada } & \multicolumn{2}{|c|}{ Frecuencia Acumulada } & \multicolumn{2}{|c|}{ Media Aritmetica de los datos } \\
\hline & $\begin{array}{c}\mathrm{N}^{\circ} \mathrm{de} \\
\text { Respuestas } \\
\mathrm{Li}\end{array}$ & $\begin{array}{c}\mathrm{N}^{\circ} \mathrm{de} \\
\text { Respuestas } \\
\text { Ls }\end{array}$ & $\begin{array}{c}\mathrm{N}^{\circ} \mathrm{de} \\
\text { Respuestas/ } \\
\mathrm{N}^{\circ} \mathrm{de} \\
\text { Expertos }\end{array}$ & $\begin{array}{c}\mathrm{N}^{\circ} \mathrm{de} \\
\text { Respuestas/ } \\
\mathrm{N}^{\circ} \mathrm{de} \\
\text { Expertos }\end{array}$ & $\begin{array}{c}\text { Suma } \\
\text { ascendente } \\
\text { de valores }\end{array}$ & $\begin{array}{c}\text { Suma } \\
\text { ascendente } \\
\text { de valores }\end{array}$ & $\begin{array}{c}\text { *Suma de los } \\
\text { datos } / 10\end{array}$ & $\begin{array}{c}\text { *Suma de los } \\
\text { datos } / 10\end{array}$ \\
\hline 0 & 264 & 222 & 0.800 & 0.673 & 1 & 1 & 1 & 1 \\
\hline 0.1 & 0 & 0 & 0 & 0 & 0.200 & 0.327 & 0.200 & 0.327 \\
\hline 0.2 & 1 & 0 & 0.003 & 0 & 0.200 & 0.327 & 0.200 & 0.327 \\
\hline 0.3 & 0 & 0 & 0 & 0 & 0.197 & 0.327 & 0.197 & 0.327 \\
\hline 0.4 & 2 & 0 & 0.006 & 0 & 0.197 & 0.327 & 0.197 & 0.327 \\
\hline 0.5 & 1 & 1 & 0.003 & 0.003 & 0.191 & 0.327 & 0.191 & 0.327 \\
\hline 0.6 & 0 & 1 & 0 & 0.003 & 0.188 & 0.324 & 0.188 & 0.324 \\
\hline 0.7 & 2 & 1 & 0.006 & 0.003 & 0.188 & 0.321 & 0.188 & 0.321 \\
\hline 0.8 & 3 & 4 & 0.009 & 0.012 & 0.182 & 0.318 & 0.182 & 0.318 \\
\hline 0.9 & 5 & 12 & 0.015 & 0.036 & 0.173 & 0.306 & 0.173 & 0.306 \\
\hline 1 & 52 & 89 & 0.158 & 0.270 & 0.158 & 0.270 & 0.158 & 0.270 \\
\hline & & & & & & & 0.187 & 0.318 \\
\hline
\end{tabular}

Fuente: Cálculos propios

* Se suman todos los datos sin tomar en consideración los valores ubicados en la casilla de cero. 
desde prácticamente humanista a cercano a humanista. Y finalmente en 2015 esta evaluación alcanzó el umbral [0.224, 0.387]. Este tipo de método de aprendizaje se puede percibir como ineficiente ya que, como se mencionó anteriormente cuando se analizó el componente recursos, las misiones educativas no cuentan con buena infraestructura y herramientas adecuadas, además este tipo de enseñanza que se centra en el individuo privilegia la atención de estudiantes menos preparados en comparación con aquellos que han sido formados en centros educativos donde se utilizan los enfoques tradicionales.

En relación con el indicador cuantitativo que describe la dimensión proceso, la normalización de este permitió identificar la totalidad de alumnos que se agrupan dentro de una misma aula de clases. De esta manera, se concluye que durante 2013 el indicador número de estudiantes por aula se explicó por un rango comprendido entre más bajo que alto el número de alumnos atendidos en función de la cantidad adecuada de estudiantes para cada aula, este resultado fue descrito por la calificación [0.412, 0.412]. En 2014 se evidenció un umbral definido por [0.588, 0.588] que describe un nivel tan bajo con alto de estudiantes por aula, identificado por el aumento en la matrícula de estudiantes durante ese año. Así mismo, en 2015 se alcanzó un umbral tan bajo como alto de estudiantes por aula explicado por una calificación de [0.412. 0.412], señal del abandonó de las aulas de clase en referido periodo (ver tabla 3 ).

Al tener todos los datos normalizados fue posible la agregación de los indicadores que explican la dimensión proceso de la educación en Mérida. A partir del método de agregación ponderada simple fueron agregados los parámetros número de estudiantes por aula, y percepción del proceso docente, de dicho procedimiento se consiguió el siguiente resultado:

$$
\begin{gathered}
\stackrel{I}{\sim}_{P}=\frac{N E+P P D}{2} \\
\stackrel{\sim}{P}_{P}=\frac{[0.412,0.412]+[0.187,0.318]]}{2} \\
{\stackrel{\sim}{I_{P}}=[0.300,0.365]}^{2}
\end{gathered}
$$

A partir de este se concluye que la eficiencia del componente proceso en el Área Metropolitana del Estado Mérida se ubicó durante 2013 en un rango cercano a ineficiente, explicado por medio de la valoración [0.300, 0.365]. En 2014 esta tendencia se conservó alcanzando un umbral equivalente a $[0.355,0.444]$ dato más ancho que el obtenido en año previo, pero que continúa representando la falta de eficiencia de las misiones educativas en el área de estudio. Para 2015 esta tendencia persistió consiguiendo un intervalo de $[0.318,0.400]$ tal como se detalla en la tabla 3.

\section{Tabla 3. Evolución del componente proceso y sus indicadores}

\begin{tabular}{|l|c|c|c|c|c|c|}
\hline \multicolumn{1}{|c|}{ Años } & \multicolumn{2}{|c|}{2013} & \multicolumn{2}{c|}{2014} & \multicolumn{2}{c|}{2015} \\
\hline $\begin{array}{l}\text { Indicador } \\
\text { de Proceso }\end{array}$ & 0.300 & 0.365 & 0.355 & 0.444 & 0.318 & 0.400 \\
\hline $\begin{array}{l}\mathrm{N}^{\circ} \text { de } \\
\text { Estudiantes } \\
\text { por Aula }\end{array}$ & 0.412 & 0.412 & 0.588 & 0.588 & 0.412 & 0.412 \\
\hline $\begin{array}{l}\text { Percepción } \\
\text { del Proceso } \\
\text { Docente }\end{array}$ & 0.187 & 0.318 & 0.121 & 0.300 & 0.224 & 0.387 \\
\hline
\end{tabular}

Fuente: Elaboración propia

\section{Avances}

Con respecto al componente avances se identificaron como indicadores objetivos: a) indicador de cobertura, 
que viene representado por el número de inscritos de cada una de las misiones educativas; y b) indicador de impacto, representado como el cociente entre el número de egresados y el número total de inscritos en cada una de las misiones educativas para el Área Metropolitana del Estado Mérida, ambos parámetros fueron normalizados mediante el método de ratios inciertos. Asimismo, se seleccionó el indicador de calidad como variable subjetiva que se expresa a través de la satisfacción de los beneficiarios que participan en los distintos programas sociales educativos, este parámetro se estandarizó a través del cálculo del expertizaje. En relación a este último procedimiento se utilizó la información disponible en la Encuesta Final de Eficiencia Educación.

Para la aplicación del expertizaje se preguntó a los beneficiarios de las misiones sociales en educación de la zona de estudio sobre los inconvenientes que se enfrentan al participar en dichas misiones, donde se obtuvieron las siguientes opiniones:

\section{¿Cuáles son los principales inconvenientes que se enfrentan al participar en dichas misiones?}

\begin{tabular}{|l|c|c|c|c|c|c|c|c|c|c|c|}
\hline & 0 & 0.1 & 0.2 & 0.3 & 0.4 & 0.5 & 0.6 & 0.7 & 0.8 & 0.9 & 1 \\
\hline $\begin{array}{l}\text { Instalación } \\
\text { Inadecuada }\end{array}$ & 237 & 0 & 0 & 0 & 0 & 2 & 0 & 2 & 0 & 8 & 81 \\
\hline $\begin{array}{l}\text { Falta de } \\
\text { Facilitadores }\end{array}$ & 230 & 5 & 3 & 4 & 3 & 3 & 3 & 1 & 8 & 8 & 62 \\
\hline $\begin{array}{l}\text { Transporte - } \\
\text { Inseguridad }\end{array}$ & 272 & 2 & 0 & 3 & 2 & 2 & 2 & 5 & 2 & 3 & 37 \\
\hline Ideología & 293 & 0 & 2 & 3 & 1 & 3 & 1 & 0 & 0 & 1 & 26 \\
\hline
\end{tabular}

Fuente: Encuesta Final de Eficiencia Educación. Elaboración propia.

El expertizaje se elaboró a partir de la representatividad de las respuestas, utilizando el planteamiento instalaciones inadecuadas (Ls) y falta de facilitadores (Li). Por medio de la utilización de ambos como intervalo de confianza fue posible identificar la percepción de los beneficiarios que hacen vida en las distintas misiones sociales educativas sobre la calidad de las mismas.

Al realizar los cálculos sobre los datos antes señalados se consiguió el siguiente resultado (Cuadro 8):

Cuadro 8. Percepción de la calidad (2013)

\begin{tabular}{|c|c|c|c|c|c|c|}
\hline Frecuencia de opiniones & \multicolumn{2}{|c|}{ Serie Normalizada } & \multicolumn{2}{|c|}{ Frecuencia Acumulada } & \multicolumn{2}{|c|}{ Media Aritmetica de los datos } \\
\hline $\begin{array}{c}\mathrm{N}^{\circ} \mathrm{de} \\
\text { Respuestas } \\
\text { Ls }\end{array}$ & $\begin{array}{c}\mathrm{N}^{\circ} \mathrm{de} \\
\text { Respuestas/ } \\
\mathrm{N}^{\circ} \mathrm{de} \\
\text { Expertos }\end{array}$ & $\begin{array}{c}\mathrm{N}^{\circ} \mathrm{de} \\
\text { Respuestas/ } \\
\mathrm{N}^{\circ} \mathrm{de} \\
\text { Expertos }\end{array}$ & $\begin{array}{c}\text { Suma } \\
\text { ascendente } \\
\text { de valores }\end{array}$ & $\begin{array}{c}\text { Suma } \\
\text { ascendente } \\
\text { de valores }\end{array}$ & $\begin{array}{c}\text { * Suma de los } \\
\text { datos } / 10\end{array}$ & $\begin{array}{c}\text { *Suma de los } \\
\text { datos } / 10\end{array}$ \\
\hline 237 & 0.697 & 0.718 & 1 & 1 & 1 & 1 \\
\hline 0 & 0.015 & 0 & 0.303 & 0.282 & 0.303 & 0.282 \\
\hline 0 & 0.009 & 0 & 0.288 & 0.282 & 0.288 & 0.282 \\
\hline 0 & 0.012 & 0 & 0.279 & 0.282 & 0.279 & 0.282 \\
\hline 0 & 0.009 & 0 & 0.267 & 0.282 & 0.267 & 0.282 \\
\hline 2 & 0.009 & 0.006 & 0.258 & 0.282 & 0.258 & 0.282 \\
\hline 0 & 0.009 & 0 & 0.248 & 0.276 & 0.248 & 0.276 \\
\hline 2 & 0.003 & 0.006 & 0.239 & 0.276 & 0.239 & 0.276 \\
\hline 0 & 0.024 & 0 & 0.236 & 0.270 & 0.236 & 0.270 \\
\hline 8 & 0.024 & 0.024 & 0.212 & 0.270 & 0.212 & 0.270 \\
\hline 81 & 0.188 & 0.245 & 0.188 & 0.245 & 0.188 & 0.245 \\
\hline & & & & & 0.252 & 0.275 \\
\hline
\end{tabular}

Fuente: Cálculos propios

* Se suman todos los datos sin tomar en consideración los valores ubicados en la casilla de cero. 
Mediante este umbral se deduce que los participantes de las misiones sociales de educación del Área Metropolitana del Estado Mérida percibieron en 2013 la calidad de dichos programas con una valoración de [0.252, 0.275], considerándose casi insatisfechos. En 2014 este umbral se modificó ampliándose el intervalo alcanzando una calificación de [0.238, 0.404] lo que significa un umbral que va desde casi insatisfechos hasta más insatisfechos que satisfechos. Para 2015 referido cálculo se estrecha nuevamente hasta [0.268, 0.293], que muestra de igual forma un nivel casi insatisfechos con la prestación de los servicios por parte de las misiones educativas.

En cuanto a los indicadores objetivos, estos se normalizaron mediante ratios inciertos, obteniéndose los siguientes resultados: el indicador de cobertura se explicó para 2013 como casi baja cobertura confirmado por la calificación [0.232, 0.232], lo que significa una tasa baja de inscripción en relación con la población objetivo a la cual se pretende beneficiar, es decir no se beneficia a tantas personas como se cree con las misiones sociales educativas. En 2014 referida variable alcanzó un umbral de [0.201, 0.201], el cual se explica como un nivel casi bajo de cobertura. Y para 2015 la banda se ubicó hasta [0.195, 0.195], lo que significa que el nivel de cobertura disminuyó en relación a los años anteriores.

Asimismo, el indicador de impacto alcanzó para 2013 un intervalo que se describe tanto de bajo impacto como de alto impacto explicado por el umbral [0.507, 0.507], esto se debe a que la Misión Robinson tuvo un alto impacto, mientras que la Misión Sucre un bajo impacto en la tasa de egreso con relación a la matricula. Para 2014 esta tendencia se mantuvo alcanzando una banda de [0.544, 0.544], que describe una situación tanto de bajo como de alto impacto de las misiones educativas en el Área Metropolitana del Estado Mérida. En 2015 la tendencia cambio, ya que se obtuvo como resultado un umbral de [0.380, 0.380], lo que describe una situación de cercano a bajo impacto, esto se explica porque los niveles de egresados de las distintas misiones educativas ha ido disminuyendo, de acuerdo a las cifras reflejadas por los entes encargados para tal fin.

Al conocer los datos que explican cada parámetro: indicador de cobertura (IC), Indicador de impacto (II), e indicador de calidad (ICA), fue posible realizar el proceso de agregación con el propósito de conocer la medida de la dimensión avances. Una vez realizado dicha agregación, mediante el uso de operaciones de matemática difusa, se obtuvieron los siguientes resultados:

$$
\begin{gathered}
{\underset{\sim}{I}}_{A}=\frac{I C+I I+I C A}{3} \\
\stackrel{\sim}{A}_{A}=\frac{[0.232,0.232]+[0.507,0.507]+[0.252,0.275]}{3} \\
{\underset{\sim}{I}}_{A}=[0.330,0.338]
\end{gathered}
$$

De estos se deduce que la eficiencia del componente avances de las misiones educativas en el Área Metropolitana del Estado Mérida se ubicó durante 2013 en un umbral de [0.330, 0.338], lo cual se describe como cercano a ineficiente. Para 2014 se colocó de igual forma en un intervalo de cercano a ineficiente que se explica por la calificación [0.328, 0.383], mientras en 2015 se alcanzó un nivel de [0.281 ,0.289]. Comparando el rango alcanzado en 2015 con los valores que explican la evaluación de los avances durante los primeros años del periodo en estudio, se concluye que es de menor magnitud, lo cual se describe como una situación de casi ineficiencia, valores que se corroboran con la información disponible en la tabla 4. 


\section{Tabla 4. Evolución del componente avances y sus indicadores}

\begin{tabular}{|l|c|c|c|c|c|c|}
\hline \multicolumn{1}{|c|}{ Años } & \multicolumn{2}{|c|}{2013} & \multicolumn{2}{c|}{2014} & \multicolumn{2}{c|}{2015} \\
\hline $\begin{array}{l}\text { Indicador } \\
\text { de } \\
\text { Avances }\end{array}$ & 0.330 & 0.338 & 0.328 & 0.383 & 0.281 & 0.289 \\
\hline Cobertura & 0.232 & 0.232 & 0.201 & 0.201 & 0.195 & 0.195 \\
\hline Impacto & 0.507 & 0.507 & 0.544 & 0.544 & 0.380 & 0.380 \\
\hline Calidad & 0.252 & 0.275 & 0.238 & 0.404 & 0.268 & 0.293 \\
\hline
\end{tabular}

Fuente: Elaboración propia

\section{Medida de eficiencia de las Misiones Educativas}

De la identificación del valor que distingue a cada indicador seleccionado para el estudio de eficiencia de las misiones educativas en el Área Metropolitana del Estado Mérida fue posible construir una medida que explica de manera plena los efectos que tiene dicha política social en los habitantes de las comunidades objeto de estudio. En este sentido, se agregaron los umbrales que describen el papel de cada uno de los indicadores a través del método de ponderación simple, en el cual todas las variables que forman el modelo pesan lo mismo dentro de la estructura de la nueva medida. En este sentido es importante resaltar que la educación deber ser estructurada por políticas que la encaminen de forma razonable, y dichas políticas del gobierno deben ser revisadas constantemente ya que de su buena estructuración se va a lograr el progreso educativo que se quiere para el país.

Las dimensiones de contexto, recursos, proceso, y avances, elegidas para explicar la eficiencia de las misiones educativas se valoraron a través de números borrosos, a los cuales se les puede aplicar los principios planteados en los operadores de matemática borrosa. A partir de la determinación del valor que distingue a cada componente seleccionado, se pudo construir el índice de eficiencia que describe la manera como las misiones educativas (Robinson, Ribas, Sucre) han influido en las condiciones de vida de los habitantes de los municipios Libertador, Campo Elías y Santos Marquina del estado Mérida, alterando el nivel de vida de sus habitantes a través del acceso a la educación, y posibilitándole la adquisición de conocimientos. Bajo este enfoque, se agregaron los umbrales que explican cada indicador para el análisis de la eficiencia de dicha misión social, obteniéndose el resultado que se muestra a continuación.

$\underset{\sim}{E}=\frac{\text { Contexto }+ \text { Recursos }+ \text { Proceso }+ \text { Avances }}{3}$

$\underset{\sim}{I E}=\frac{[0.264,0.452]+[0.159,0.159]+[0.300,0.365]+[0.330,0.338]}{4}$

A partir de los valores obtenidos se desprende que para 2013, la eficiencia de las misiones educativas en el Área Metropolitana del estado Mérida se ubicó en un nivel entre casi ineficiente hasta cercano a ineficiente, es decir se identificó en el umbral [0.263, 0.329]. Con esta explicación se evita el análisis de la eficiencia de las políticas públicas solo desde la óptica binaria que describía la existencia únicamente de alta o baja eficiencia, y se utilizan ahora intervalos de confianza que aumentan la probabilidad de que un hecho ocurra, describen todas las alternativas posibles entre dos condiciones, y toman en cuenta el dato real con toda la entropía presente en la realidad. En 2014, el efecto neto de las misiones educativas sobre los habitantes del Área Metropolitana del Estado Mérida se explicó a través de un nivel entre casi ineficiente hasta cercano a ineficiente, contenido en el umbral [0.291, 0.355]. Finalmente, para 2015 la eficiencia de los 
programas socioeducativos en el Área Metropolitana del Estado Mérida se reconoció en el nivel casi ineficiente hasta cercano a ineficiente, igual que en los años previos, con la particularidad de que en este último periodo los límites del umbral se aproximan entre sí cerrando la banda hasta los valores $[0.254,0.324]$ (ver tabla 5).

\section{Tabla 5. Evolución del índice de eficiencia y sus indicadores}

\begin{tabular}{|l|c|c|c|c|c|c|}
\hline Años & \multicolumn{2}{|c|}{2013} & \multicolumn{2}{c|}{2014} & \multicolumn{2}{c|}{2015} \\
\hline $\begin{array}{l}\text { Índice de } \\
\text { Eficiencia } \\
\text { (IE) }\end{array}$ & 0.263 & 0.329 & 0.291 & 0.355 & 0.254 & 0.324 \\
\hline $\begin{array}{l}\text { Indicador de } \\
\text { Contexto }\end{array}$ & 0.264 & 0.452 & 0.322 & 0.436 & 0.272 & 0.461 \\
\hline $\begin{array}{l}\text { Indicador de } \\
\text { Recurso }\end{array}$ & 0.159 & 0.159 & 0.158 & 0.158 & 0.145 & 0.145 \\
$\begin{array}{l}\text { Indicador de } \\
\text { Proceso }\end{array}$ & 0.300 & 0.365 & 0.355 & 0.444 & 0.318 & 0.400 \\
\hline $\begin{array}{l}\text { Indicador de } \\
\text { Avances }\end{array}$ & 0.330 & 0.338 & 0.328 & 0.383 & 0.281 & 0.289 \\
\hline
\end{tabular}

Esta situación de ineficiencia en los programas socioeducativos se expresa a causa del reducido nivel de los indicadores de contexto y recursos, valores que se encuentran por debajo del nivel final del índice de eficiencia, manifestándose como una insuficiencia en la dotación de los recursos materiales y humanos indispensables para su debido funcionamiento, teniendo como consecuencia una falta de transparencia en el uso de los recursos públicos. A parte de ello, Como puede observarse las misiones educativas no son tan masivas como se piensa, ya que presentan un bajo nivel de cobertura en la población que se desea atender y esto se debe básicamente a la utilización de estos programas como instrumento de adoctrinamiento ideológico, de manera que resultan excluyentes hacia quienes no se identifican con el proceso revolucionario. Significa entonces que las misiones educativas se caracterizan por su baja eficiencia, es decir, que estos programas no se están ejecutando tal como está establecido en su diseño, en consecuencia no están cumpliendo con los objetivos definidos, siendo uno de ellos la reducción de la pobreza por medio de la capacitación de la población, a su vez problemas como el analfabetismo, la deserción escolar, la capacitación para el trabajo siguen presentes.

\section{Conclusiones}

La utilización de lógica difusa en el campo de la ciencia social proporciona la capacidad de modelar el comportamiento de los distintos agentes económicos ya que permite incorporar en el análisis las ambigüedades e imprecisiones del ser humano. En este sentido, la lógica difusa permitió la valoración de indicadores objetivos y subjetivos útiles para explicar la eficiencia de las misiones educativas del gobierno venezolano en el Área Metropolitana del Estado Mérida. Una de las ventajas que ofreció la lógica difusa, fue la capacidad de trabajar tanto con información cuantitativa como con opiniones de las personas; esta última propiedad permitió un tratamiento matemático formal de la información vaga, subjetiva y difícil de medir que existe en la realidad.

Con la aplicación del método de ratios inciertos, se logró la transformación de forma sencilla a una unidad homogénea de los distintos valores obtenidos por los indicadores cuantitativos seleccionados para el estudio de la eficiencia de los programas socioeducativos. Mediante la técnica de expertizaje se logró identificar y usar variables cualitativas dentro del análisis de eficiencia de mencionada política social, lo cual permitió convertir la información subjetiva a la misma escala referida para las variables objetivas. Desde 
esta perspectiva se logró un análisis de la eficiencia de las misiones sociales en educación por medio de la información objetiva y la información que contiene la percepción del individuo sobre su participación en estos programas.

La agregación de los resultados obtenidos para cada uno de los indicadores: contexto, recursos, proceso y avances, permitió identificar la eficiencia de las misiones educativas en el área en estudio mediante un índice de eficiencia. Dicha medida se representó a través de un umbral de confianza, constituyéndose en distintos niveles de eficiencia alcanzados durante el periodo en estudio, dando lugar de esta manera a la evaluación integral de los efectos generados por dicha política social en la población de los municipios Libertador, Campo Elías y Santos Marquina del estado Mérida.

En este sentido, el índice de eficiencia de las misiones educativas para los años 2013, 2014 y 2015 dio un intervalo de confianza de [0.263, 0.329], [0.291, 0.355], [0.254, 0.324] respectivamente, observándose una tendencia en el periodo en estudio, que esta representada por una clasificación de casi ineficiente a cercana a ineficiente. Esto debido principalmente al reducido nivel de los indicadores de recursos, que se puede reflejar como una insuficiencia en la dotación de los recursos materiales y humanos indispensables para el debido funcionamiento de las misiones Robinson, Ribas y Sucre, así como por el hecho de que el espacio físico donde se llevan a cabo los programas no cuenta con las condiciones necesarias para instrumentarlos, teniendo en cuenta que la metodología educativa de los programas socioeducativos está basada en el sistema de video-clases como método de enseñanza, ayudado por la orientación de un facilitador. Por otro lado, los resultados describen que estas misiones han logrado tener una gran aceptación por parte de la población, ya que a pesar de que todos los programas educativos presentan limitaciones en su implementación, han permitido incorporar al sistema educativo a las personas que no pudieron estudiar antes, garantizando la inclusión de alguna manera de las personas excluidas, sin embargo, estos programas han sido utilizados como instrumento de adoctrinamiento ideológico, resultando excluyentes hacia quienes no se identifican con el proceso revolucionario. Otra de las razones que explica la ineficiencia de las misiones educativas es el ofrecimiento de una educación de calidad que no parece estar lográndose por los distintos inconvenientes que se presentan en el aula de clase, siendo uno de ellos la falta de facilitadores que se comprobó por las opiniones emitidas por los participantes de dichos programas.

\section{REFERENCIAS BIBLIOGRÁFICAS}

D’Elias, Y. y Cabezas, L. (2008) Las Misiones Sociales en Venezuela. Ildis (Instituto Latinoamericano de Investigaciones Sociales). Caracas-Venezuela.

Gil, J. (1999). Teoría de la Incertidumbre en el Ámbito Económico. SEPEC- ULA: Mérida.

Gómez, I. (2007). El papel de las misiones sociales en la construcción de identidades políticas en Venezuela. Revista Venezolana de Economía. Y Ciencias Sociales, vol. 13, $n^{\circ} 1$ (ene.abr.), pp. 13-34. Recuperado en: http://www.scielo.org.ve/scielo.php?script=sci_arttext\&pi$\mathrm{d}=$ S131564112007000100002\&lng=es\&tlng=es 
Hurtado, A. y Tinto, J. (2009). Nueva técnica para medir la pobreza utilizando la teoría de la incertidumbre. Revista Economía, Núm. 28, julio-diciembre, pp. 213-237. ISSN 1315-2467.

Hurtado, A.; Tinto, J. y Zerpa, S. (2011). Medición de la calidad de vida en Mérida a través de la lógica difusa. Revista Economía, Núm. 32, julio-diciembre, pp. 67-94. ISSN 1315-2467.

Hurtado, A y Zerpa, S. (2016). Misiones sociales en Venezuela: concepto y contextualización. Revista Sapienza Organizacional, Núm. 6, julio-diciembre, pp. 37-64. ISSN 2443-4256.

Kaufmann, A. y J. Gil (1993). Técnicas especiales para la gestión de expertos. Santiago de Compostela: Editorial Milladoiro, $376 \mathrm{pp}$.

López, M. y Gentile, N. (2008). Sistema de indicadores económicos y sociales: la importancia del análisis integrado. Presentado en Encuentro Nacional de la Red de Economías Regionales en el Marco del Plan Fénix, 9. Jornadas Nacionales de Investigadores de las Economías Regionales, 2, Tandil, 18-19 septiembre. Recuperado en: http://nulan.mdp.edu.ar/1037/\#.VNzNDeaG8rW

Picado, X. (1997) Hacia la elaboración de indicadores de evaluación. Recuperado en: http://www. ts.ucr.ac.cr/binarios/docente/pd-000088.pdf

Quesada, A. (2010) Caos, complejidad e incertidumbre en los movimientos bursátiles de las empresas energéticas (hidrocarburos) en el mercado de valores internacional. Tesis doctoral para optar al título de Doctora en Ciencias Económicas, Universidad del Zulia- Venezuela.

Zadeh, L.A. (1965) “Fuzzy set”, Information and Control, 8, pp. 338-353.

\section{Para citaciones:}

Contreras-Guillén, D. D., \& Hurtado-Briceño, A. J. (2017). Eficiencia de las misiones sociales de educación utilizando lógica difusa. Panorama Económico, 25, 1, pp. $19-42$.

\section{AUTORES}

Darwin Diskey Contreras-Guillén

Profesor Instructor del Departamento de Economía, Facultad de Ciencias Económicas y Sociales (FACES) de la Universidad de los Andes (ULA) en la Republica Bolivariana de Venezuela. Economista y Magíster en Economía con mención en Economía Cuantitativa de la misma institución.

Alberto José Hurtado-Briceño

Profesor Agregado del Departamento de Economía de la Facultad de Ciencias Económicas y Sociales (FACES) de la Universidad de los Andes (ULA) en la Republica Bolivariana de Venezuela. Actualmente es Editor de Revista Economía (ULA) y Coordinador del Grupo de Gestión Económica en la Incertidumbre (GEIN) del Instituto de Investigaciones Económicas y Sociales de la Universidad de los Andes (Venezuela). En la actualidad cursa estudios doctorales en Ciencias Humanas en la Universidad de los Andes (Venezuela), y es Magíster en Economía con mención en Políticas Económicas (Universidad de Los Andes, Venezuela). Sus Líneas de investigación corresponden el analisis de Pobreza, la Calidad de Vida, Lógica Difusa, Sistemas Agroalimentarios, Integración Económica, Integración Monetaria y Política Monetaria. 


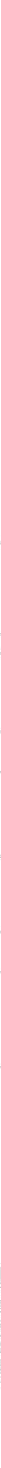

